\title{
Sentimental Analysis by Speech-Video Recognition using Machine Learning
}

\author{
Ms.Nikita Gavhane ${ }^{1}$, Ms.Sayali Kolte ${ }^{2}$, Ms. Smita Botre ${ }^{3}$, Prof.Avinash Palave ${ }^{4}$ \\ Student, Computer Department, KJEI's Trinity College of Engineering and Research Pisoli, Pune, India ${ }^{1,2,3}$ \\ Guide, Computer Department, KJEI's Trinity college of Engineering and Research Pisoli, Pune, India ${ }^{4}$
}

\begin{abstract}
Presently large amount of data is available on social networking sites, product review sites, blogs, forums etc. This data holds expressed opinions and sentiments. The volume, variety, velocity are properties of data, whether it comes from the Internet or an enterprise resource planning system, sentiment analysis system should get the data and analyze it. Due to the large volume of opinion rich web resources such as discussion forum, review sites, blogs and news corpora available in digital form, much of the current research is focusing on the area of sentiment analysis and opinion mining. Expression of any sentiment is a mixture of text, prosody, facial expression, body posture etc. Thus only text input cannot fully represent a sentiment. A multimodal system uses a combination of input modes e.g. text and audio or text and video or all of these three. This paper analyses the techniques used for sentiment data and also demonstrate that how individual model works. Extracting the sentiments from different input modes is achieved by different classifying techniques. The proposed method first digitizes the signal to extract the required properties. According to emotional Prosody studies, the tone of every person's voice can be characterized by its pitch, loudness or intensity, timbre, speech rate and pauses, whose changes convey different information from the speaker to the listener
\end{abstract}

Keywords: Sentiments, Emotion, Video, Audio.

\section{INTRODUCTION}

Opinion Mining is about "What the other people think" that has always been an important piece of information for most of the users during the decision-making process. The awareness of the World Wide Web (WWW) became widespread, many among us asked our friends to recommend an auto mechanic, to explain who they were planning to vote for in local elections, request for reference letters regarding job application from colleagues, or consulted consumer reports to decide what dishwasher is best to buy. But now the web and the Internet have (among other things) made it possible to find out about the opinions and experiences of those among the vast pool of people that are neither our personal acquaintances. Speech recognition is the process of converting spoken language to written text or some similar formats. The major steps of a typical speech recognizer are as follow: At first, design of grammar which is the process of recognition of grammars which define the words that may be spoken by a user and the patterns in which they may be spoken. A grammar must be created and activated for a recognizer to know what it should listen for in incoming audio, signal processing which is used to analyse the frequency characteristics of the incoming audio, phoneme recognition is the process of comparing the spectrum patterns to the patterns of the phonemes of the language being recognized, word recognition is the process of comparing the sequence of likely phonemes against the words and patterns of words which specified by the active grammars, result generation provides the application along with information about the words that the recognizer has detected in the incoming audio. However, the sentimental analysis is also practical for use in business analytics and situations in which text needs to be analyzed.

\section{LiteratURE SURVEY}

"A survey of sentiment analysis techniques",

Harpreet Kaur ; Veenu Mangat ; Nidhi, in: 2017 International Conference on I-SMAC (IoT in Social, Mobile, Analytics and Cloud) (I-SMAC)[1]

Sentiment analysis is an application of natural language processing. It is also known as emotion extraction or opinion mining. This is a very popular field of research in text mining. The basic idea is to find the polarity of the text and classify it into positive, negative or neutral. It helps in human decision making. To perform sentiment analysis, one has to perform various tasks like subjectivity detection, sentiment classification, aspect term extraction, feature extraction etc. This paper presents the survey of main approaches used for sentiment classification. 
Vol. 8, Issue 4, April 2019

\begin{abstract}
"A NOVEL VOICE BASED SENTIMENTAL ANALYSIS TECHNIQUE TO MINE THE USER DRIVEN REVIEWS”, International Research Journal of Engineering and Technology (2017)[2]

Sentimental analysis plays a vital role now-adays because many start-ups have been emerged based on user-driven content. Many service-based organizations are basically user opinion based online agents rendering services to consumers. The proposed method helps to convert speech review into text based on speech recognition module. The user reviews (text) are stored in cloud for audit purpose. Once the audit is performed the reviews are posted in the respective applications. In these user-driven reviews about a product is taken into sentimental analysis to get positive, negative and neutral words. This would make the consumer come to a decision in a fraction of a section rather than going through number of reviews, thus tremendously saving time. Our main contributions include a voice-based trust model for computing user feedback comments. The proposed system involves machine learning language for classification and assigning weightage to each positive, negative and neutral word. The proposed method scaled well for different types of opinion
\end{abstract}

Twitter as a Corpus for Sentiment Analysis and Opinion Mining Alexander Pak, Patrick Paroubek Universit'e de Paris-Sud, Laboratoire LIMSI-CNRS, B`atiment 508, F-91405 Orsay Cedex, France alexpak@limsi.fr, [3]

Microblogging today has become a very popular communication tool among Internet users. Millions of users share opinions on different aspects of life everyday. Therefore microblogging web-sites are rich sources of data for opinion mining and sentiment analysis. Because microblogging has appeared relatively recently, there are a few research works that were devoted to this topic. In our paper, we focus on using Twitter, the most popular microblogging platform, for the task of sentiment analysis. We show how to automatically collect a corpus for sentiment analysis and opinion mining purposes. We perform linguistic analysis of the collected corpus and explain discovered phenomena. Using the corpus, we build a sentiment classifier, that is able to determine positive, negative and neutral sentiments for a document. Experimental evaluations show that our proposed techniques are efficient and performs better than previously proposed methods. In our research, we worked with English, however, the proposed technique can be used with any other language.

\title{
III. PROPOSE SYSTEM
}

Sentiment analysis is a method, which classifies the given data due to having positive or negative opinion and being subjective or objective in general.

We propose android and web based application which construct sentiments analysis system. The main module in this system are:-

1. User:-In this user get registered with system using unique id and password .

2. Admin:-Admin is responsible for adding user, authenticate user and managing other module and managing dataset. 3.System:-In this proposed system user will upload images, video ,audio he/she want .Once its uploaded it get classify by system and get analyzed and display result to user.

4.All data get stored in database of system.

\section{IV.SYSTEM ARCHITECTURE}

Following diagram is our system's architecture diagram:

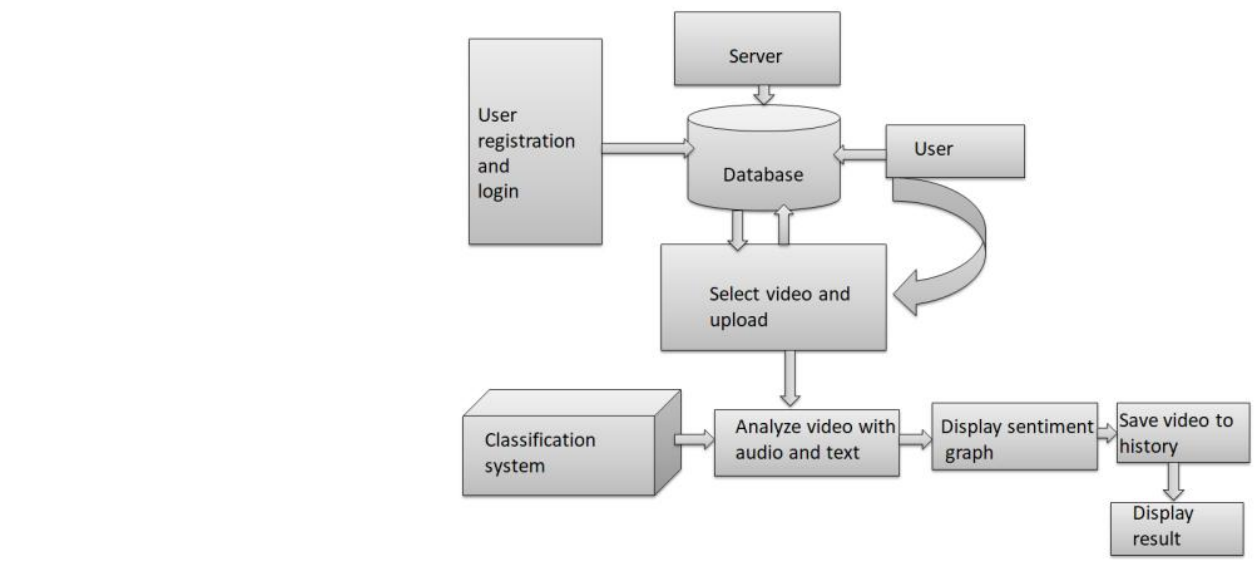

- User Feature:

Figure 1: System Architecture

1. Upload video.

2. User Profile. 


\section{International Journal of Advanced Research in Computer and Communication Engineering}

Vol. 8, Issue 4, April 2019

3. Record audio.

4. Basic user functions like history change password.

- $\quad$ Admin Feature:

5. Admin profile.

6. Manage users.

7. Manage authentication.

8. Manage dataset of keywords.

9. Train dataset according to video datasets.

Other Non- functional requirements.

\section{Methodologies}

\section{Mathematical Model}

Let $\mathrm{S}$ be the Universal Final Set :

$\mathrm{S}=\{\mathrm{s}, \mathrm{e}, \mathrm{v}, \mathrm{i}, \mathrm{o}$, functions, DD, NDD, Success, Failure $\}$ Where,

$\mathrm{s}=$ initial state $=$ Upload video

$\mathrm{e}=$ end state $=$ sentiment analysis $\mathrm{v}=$ video 1, video $2, \ldots \ldots$, videoN $\mathrm{i}=$ Input $=\mathrm{v}$

$\mathrm{o}=$ output $=$ Graph functions $=\mathrm{f} 1, \mathrm{f} 2, \mathrm{f} 3$

$\mathrm{f} 1=$ Video to audio conversion

$\mathrm{f} 2=$ Audio to text conversion

$\mathrm{f} 3=$ Text analysis to get sentiments

Deterministic data $=$ same output from a given starting condition and return the same result any time

Non deterministic data $=$ result will vary every time for given input Success condition = According to proper inputs, sentiment will get analyze

failure condition $=$ Wrong inputs and ambiguity in audio of video.

\section{AlgORITHM}

\section{SVM algorithm}

Step 1: Consider data set

Step 2: Design hyperplane

Step 3: Hyperplane divides training vectors into two classes i.e. positive and negative Positive class contains

Step 4: Result sentiments like love , happy ,joy, excite Negative class contains sentiments like sad, hate, poor, sorrow

\section{BEYOND VERBAL API}

The Beyond Verbal Emotions Analytics API programmatically understands and analyzes emotions for the developer's voice-powered device, system, or app. This service can determine a recorded voice's valence, arousal, temper, and mood group. Valence indicates overall positivity or negativity. Arousal measures the speaker's level of alertness or stimulation. Temper is a metric that covers the speaker's entire mood range, from depressed to friendly to aggressive. Mood groups indicate the speaker's emotional state and include anger, loneliness, happiness, and excitement, among others.

\section{CONCLUSION}

In this proposed system is android application

Sentiment Analysis problem is a machine learning problem that has been a research interest for recent years. Though lot of work is done till date on sentiment analysis, there are many difficulties to sentiment analyser since Cultural influence, linguistic variation and differing contexts make it highly difficult to derive sentiment. The reason behind this is unstructured nature of natural language

This paper investigated the prediction of the next reactions from emotional vocal signals based on the recognition of emotions, using different categories of classifiers.. The leadership, anger, disagree, self-control emotions were chosen. Eleven spectral features, namely, pitch, intensity, the first four formants and their bandwidths and standard deviation, were extracted. 


\title{
International Journal of Advanced Research in Computer and Communication Engineering
}

\author{
Vol. 8, Issue 4, April 2019
}

\section{FUTURE WORK}

The field of sentiment analysis is an exciting new research direction due to large number of real-world applications where discovering people's opinion is important in better decision-making. The development of techniques for the document-level sentiment analysis is one of the significant components of this area. Recently, people have started expressing their opinions on the Web that increased the need of analyzing the opinionated online content for various real-world applications. A lot of research is present in literature for detecting sentiment from the text. Still, there is a huge scope of improvement of these existing sentiment analysis models. Existing sentiment analysis models can be improved further with more semantic and commonsense knowledge

\section{RESULT}
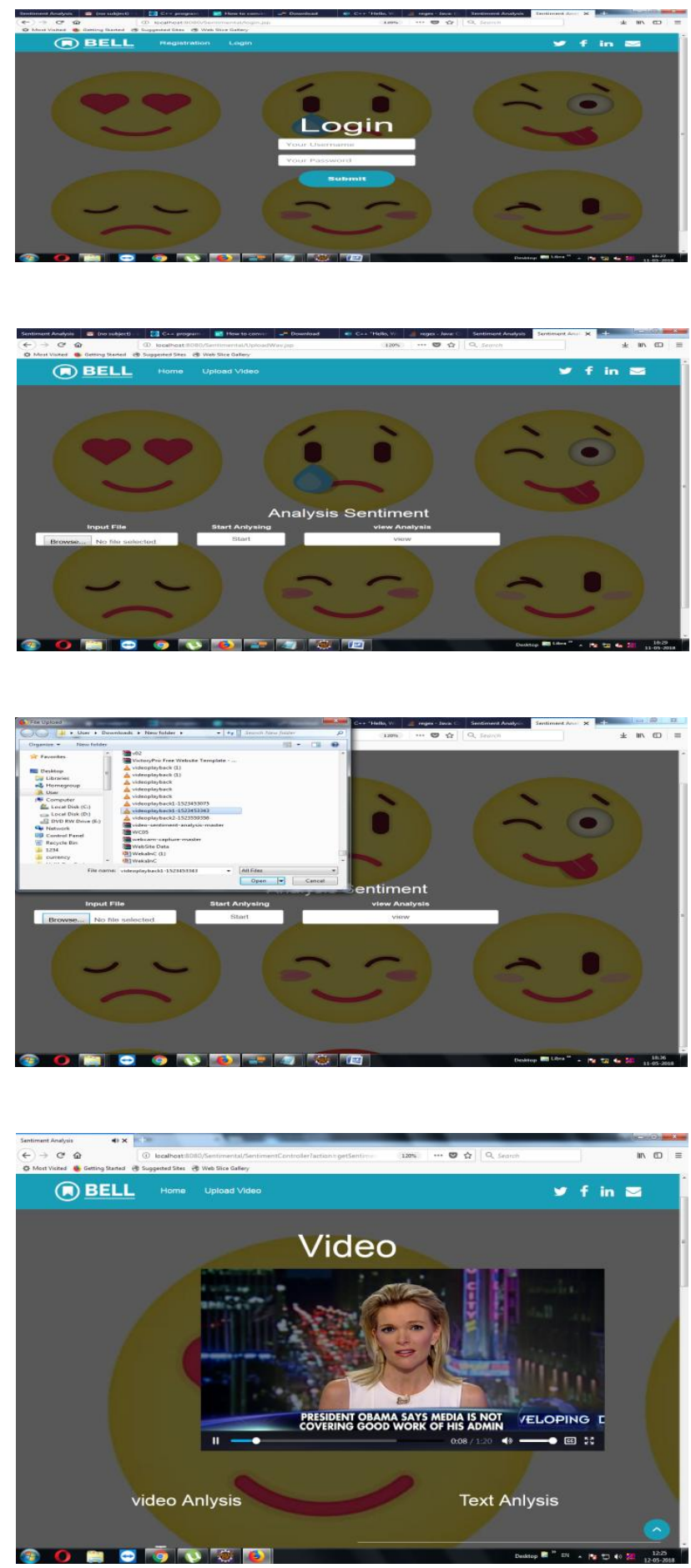


\section{International Journal of Advanced Research in Computer and Communication Engineering}

Vol. 8, Issue 4, April 2019

\section{IX.GRAPH}

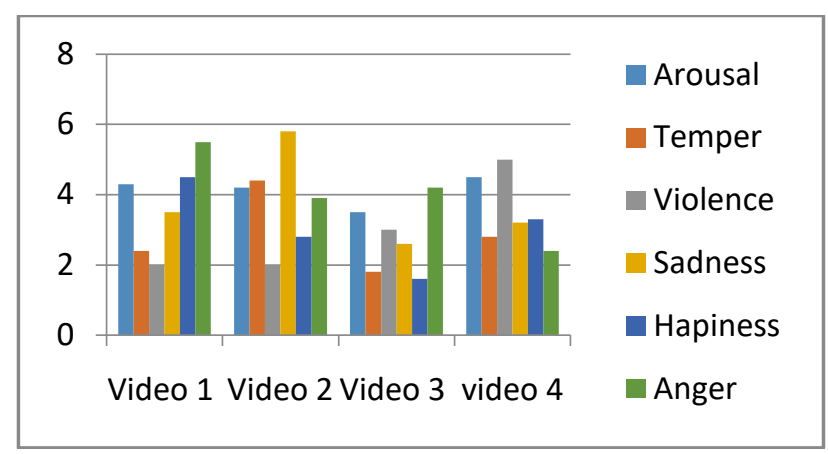

\section{REFERRENCES}

[1]. A survey of sentiment analysis techniques", Harpreet Kaur ; Veenu Mangat ; Nidhi, in: 2017 International Conference on I-SMAC (IoT in Social, Mobile, Analytics and Cloud) (I-SMAC)

[2]. “A NOVEL VOICE BASED SENTIMENTAL ANALYSIS TECHNIQUE TO MINE THE USER DRIVEN REVIEWS”, International Research Journal of Engineering and Technology (2017)

[3]. Biologically inspired speech emotion recognition

[4]. Aishwarya Murarka, Kajal Shivarkar, Sneha, Vani Gupta, Prof.Lata Sankpal, Sentiment Analysis of Speech, International Journal of Ad- vanced Research in Computer and Communication Engineering, 11, November 2017.

[5]. Erik Boiy, Marie-Francine Moens, A Machine Learning Ap- proach to Sentiment Analysis in Multilingual Web Texts, August 13, 2008.

[6].Svetlana Kim, YongIk Yoon, Fusion Sentimental Analysis in Self-Growth Broadcasting, 2018 IEEE International Conference on Big Data and Smart Computing.

[7].Lakshmish Kaushik, Abhijeet Sangwan, John H L. Hansen, Sen- timent extraction from natural audio streams,2018 IEEE International Conference on Big Data and Smart Computing.

[8].G, Castellano, L. Kessous, G. Caridakis, G Emotion recognition through multiple modalities: Face, body gesture,speech, affect and emotion in humancomputer interaction, In C. Peter amp; R. Beale (Eds.), Lecture notes incomputer science 4868 (pp. 92103 ). [Berlin Heidelberg: Springer. 2008.

[9].M. Wollmer, F. Weninger, T. Knaup, B. Schuller, C. Sun, K. Sagae, L.P. Morency, Youtube movie reviews: Sentiment analysis in an audiovisual context, Intell. Syst. IEEE, 28 (3) (2013), pp. 46-53.

[10].Bin Wen, Shanrong Duan , Bin Rao , Wenhua Dai, Research on Word Sentimental Classification based on Transductive Learning, 2015 8th International Symposium on Computational Intelligence and Design.

[11].Martin Wöllmer, Felix Weninger, Tobias Knaup, and Björn Schuller, Technische Universität München,Congkai Sun, Shanghai Jiaotong university,Kenji Sagae and Louis-Philippe Morency, University of Southern California, You-tube movie Reviews: Sentiment Analysis is an Audio Visual Context ,2013 IEEE 